\title{
Immune Reactive Ezrin Surface Area Increases in Glomerular Podocytes of STZ Diabetic Rats Precede Their Detachment, Is Prevented by Phlorizin But Not by Insulin
}

\author{
Slava Malatiali', Issam Francis ${ }^{2}$, Musleeha Chesor ${ }^{3}$, Gavin Welsh ${ }^{3}$, Moin Saleem ${ }^{3}$, \\ Mario Barac-Nieto1,4 \\ ${ }^{1}$ Departments of Physiology, Faculty of Medicine, Kuwait University, Safat, Kuwait \\ ${ }^{2}$ Pathology, Faculty of Medicine, Kuwait University, Safat, Kuwait \\ ${ }^{3}$ University of Bristol, Pediatric Nephrology, Bristol, UK \\ ${ }^{4}$ Retired 9443 Hickory Limb, Colusmbia MD, USA \\ Email: mario.baracnieto @gmail.com
}

How to cite this paper: Malatiali, S., Francis, I., Chesor, M., Welsh, G., Saleem, M. and Barac-Nieto, M. (2020) Immune Reactive Ezrin Surface Area Increases in Glomerular Podocytes of STZ Diabetic Rats Precede Their Detachment, Is Prevented by Phlorizin But Not by Insulin. Journal of Diabetes Mellitus, 10, 27-40.

https://doi.org/10.4236/jdm.2020.102003

Received: February 15, 2020

Accepted: March 28, 2020

Published: March 31, 2020

Copyright $\odot 2020$ by author(s) and Scientific Research Publishing Inc. This work is licensed under the Creative Commons Attribution International License (CC BY 4.0).

http://creativecommons.org/licenses/by/4.0/

\begin{abstract}
Glomerular tuft immune reactive Ezrin surface area (EzA) and fraction of EzA to total glomerular tuft area significantly increased, indicating podocyte growth, rounding and altered cytoskeletal interactions at 1 week of STZ diabetes. Podocyte number per glomerulus (WT1+ nuclei) did not change indicating no detachment, but density decreased due to tuft hypertrophy. Treatment with PLZ or Insulin for one week, prevented increase in proteinuria and hyperglycemia but not the decrease in podocyte density. PLZ but not Insulin prevented increase in ezrin positive area in glomeruli and per podocyte. In podocytes in culture neither $25 \mathrm{mM}$ glucose with or without PLZ (2.5 or $25 \mathrm{uM}$ ) altered Ezrin expression measured in western blots. In summary, the Ezrin positive glomerular surface area increase seen after 1 week of STZ diabetes, reflects altered podocyte morphology and cytoskeletal interactions, prevented by PLZ but not by insulin. Ezrin area increase preceded podocyte detachment and in podocytes in culture is not associated with increases in podocyte Ezrin protein expression. It is a likely precursor of shape changes in podocytes and of alterd interactions with basement membrane that contribute to detachment and thickening. Glomerular capillary tuft hypertrophy and reduced podocyte density persisted despite PLZ or insulin treatments, independently of levels of glycemia and of proteinuria.
\end{abstract}

\section{Keywords}

Diabetes. Hyperglycemia, Glomerular Hypertrophy, Phlorizin, Podocyte 
Density, Ezrin

\section{Introduction}

Podocytes are the major cells responsible for selectivity of the glomerular filtration barrier [1] and are the target of many pathophysiological processes resulting in glomerular disease. Their complex and delicate architecture, wrapping around glomerular capillaries and defining the filtration slit diaphragms plays a critical role in determining the perm-selectivity of the glomerular barrier [2].

In diabetes mellitus, glomerular pathology is a main and early result of the microvascular component of the disease, often translating in hypertrophy, hyperfiltration and micro albuminuria [3] and evolving into glomerulosclerosis, overt proteinuria and renal failure [4].

Normalization of blood glucose is a major but difficult goal to achieve during treatment of diabetes which has recently been approached with the use of oral analogues of phlorizin, inhibitors of $\mathrm{Na}^{+}$-Glucose cotransporter (SGLT2), as adjutants in diabetes therapy [5] and effective at reducing morbidity and mortality in diabetics [5].

Previous studies indicate that alleviating hyperglycemia with phlorizin prevented the development of hyperfiltration and proteinuria despite persistent glomerular capillary tuft hypertrophy [6].

In the present study, structural changes in glomerular podocytes early during diabetes and the effects of phlorizin and insulin on these changes are evaluated. In particular, ezrin, a cytoskeletal-membrane linker protein involved in epithelial cell morphogenesis, adhesion and signal transduction [7] and of Wilms tumor-1 a protein typical of podocyte nuclei [8] were measured by immunohistochemistry to assess changes in the podocyte membrane-cytoskeleton and in their number, respectively, in diabetic and phlorizin or insulin-treated diabetic rats. Ezrin expression was measured in western blots of podocytes in vitro exposed to 5 or $25 \mathrm{mM}$ glucose and with or without PLZ.

Increase in the glomerular podocytes ezrin positive area was found to be reversed by PLZ but not by insulin, while proteinuria and hyperglycemia were blunted by both treatments [6]. No changes in Ezrin expression were found in the cultured podocytes.

\section{Materials and Methods}

1) Animals

Male Fischer rats (8 weeks old) were kept in a room with an 8:00 - 20:00 light, 20:00 - 8:00 dark cycle, at $22.3^{\circ} \mathrm{C} \pm 0.3^{\circ} \mathrm{C}$ and $31.2 \% \pm 0.8 \%$ humidity. Rats had free access to water and standard rat chow. All animals were cared for in accordance with the Guide for the Care and Use of Laboratory Animals and all experimental protocols were approved by the Research Administration at Kuwait 
University. Five groups were used: Control rats (C), diabetic rats (D), diabetic rats treated with insulin (DI) or Phlorizin (DPLZ), and diabetic rats treated with propylene glycol, the vehicle for PLZ (DPG).

2) Induction of diabetes and treatments

Diabetes was induced by intra-peritoneal (i.p) injection of $55 \mathrm{mg} / \mathrm{kg}$ Streptozotocin (STZ, S-0130, Sigma, USA), dissolved in $55 \mathrm{mM}$ trisodium-citrate buffer, $\mathrm{pH}=4.5$. Controls received the citrate buffer (vehicle) alone. After STZ injection, animals were placed in metabolic cages and development of diabetes was confirmed 16-hrs later by measuring blood glucose concentration from samples taken from the tail vein and using a gluco-meter (GLUCOTREND 2, Roche, Germany).

3) Phlorizin treatment

Phlorizin is a well-known inhibitor of renal glucose reabsorption that interacts mainly with the SGLT2 type of glucose transporters. Recently, a number of its derivatives that can be given orally have been developed and are being extensively used in the treatment of diabetes. Diabetic rats (DPLZ) were treated with phlorizin (P-3449, Sigma, USA), $400 \mathrm{mg} / \mathrm{kg} /$ day, starting $16 \mathrm{hrs}$ after STZ injection, as described earlier [6]. Vehicle treated diabetic rats (DPG) were given 0.1 $\mathrm{ml}$ propylene glycol. Blood glucose levels were measured twice daily in PLZ-treated and diabetic rats. At day 7 before animal sacrifice; fasting blood glucose levels were measured in all groups, using Glucotrend 2 Roche.

4) Insulin Treatment.

Diabetic rats were treated with insulin (DI), (Mixtard 30 HM, Novo Nordisk, Denmark) (30\%, 70\%, $100 \mathrm{IU} / \mathrm{ml})$, starting twenty-four hours after STZ injection. The rats were given subcutaneous (s.c.) injection of $1-2$ units of insulin (dissolved in $0.1-0.3 \mathrm{ml}$ of sterile $0.9 \% \mathrm{NaCl}$ ) at 8:00 p.m. just before feeding and $0.5-1$ units at 8:00 a.m. when needed, for six days.

5) Protein Excretion Rate.

Protein excretion rate (PER) was calculated from urinary protein concentration $(\mathrm{Up}, \mathrm{mg} / \mathrm{ml})$ and urine volume collected in metabolic cages for $24 \mathrm{~h}(\mathrm{ml} / 24$ hrs), as previously described [6].

6) Perfusion fixation of rat kidneys.

Rat left kidneys were perfusion-fixed with $10 \%$ neutral-phosphate buffered formalin, embedded in wax, cut into 5-micron thick sections and mounted on APES-coated slides for immuno-histochemical studies.

7) Immunohistochemistry.

Kidney tissue sections were immunostained with two antibodies to podocyte markers: a) Rabbit polyclonal anti-ezrin antibody (Upstate Biotechnology, USA), against a synthetic peptide corresponding to amino acids 479 - 498 of human ezrin, which is $17 / 20$ amino acids identical to those of rat ezrin and is specific for the $81 \mathrm{kDa}$ ezrin, a podocyte cytoskeletal marker [7]. b) Rabbit polyclonal anti-Wilm's tumor-1 antibody (WT-1, Sigma, USA), which is specific for the 52 $\mathrm{kDa}$ Wilms' tumor nuclear protein exclusively expressed in nuclei of podocytes in the glomerulus [9]. 
The immunostaining procedure used was the LSAB2 system from DACO ( $\mathrm{K}$ 0609, Denmark) using a biotinylated anti-rabbit antibody and streptavidin-conjugated horseradish peroxidase (HRP), especially developed for rat tissues to minimize background and cross-reactivity. The substrate for the HRP enzyme was di-aminobenzidine (DAB, Denmark).

8) Immunostain with Anti-ezrin Antibody.

The sections were hydrated, and then treated with $3 \% \mathrm{v} / \mathrm{v} \mathrm{H}_{2} \mathrm{O}_{2}$ for $10 \mathrm{~min}$ to inhibit endogenous peroxidase activity. For antigen retrieval, sections were placed in $11 \mathrm{mM} \mathrm{Na}^{+}$-citrate buffer, $\mathrm{pH}=6$ and heated in a microwave oven at 950 watts for $10 \mathrm{~min}$.

The sections were then blocked with 10\% v/v goat serum (DACO, Denmark) for $15 \mathrm{~min}$, and then incubated with anti-ezrin antibody (diluted 1:50) overnight at $4^{\circ} \mathrm{C}$ in a hydrated chamber. After washing with tris-buffered saline (TBS: $\mathrm{NaCl}$ : 8.1 g, Tris: $0.6 \mathrm{~g}, 4.2 \mathrm{ml} \mathrm{1M} \mathrm{HCl}$ per liter, $\mathrm{pH}=7.6$ ), $100 \mu \mathrm{l}$ of the secondary biotinylated antibody reagent were added on each section for $30 \mathrm{~min}$, washed again with TBS, and then exposed to $100 \mu \mathrm{l}$ of streptavidin-HRP reagent for another $30 \mathrm{~min}$. Finally, $100 \mu \mathrm{l}$ of DAB $(1 \mathrm{mg} / \mathrm{ml}$ in TBS) were added to the sections.

9) Immunostain with Anti-WT-1 antibody.

The immunostaining procedure followed with the anti WT-1 antibody was similar to that described for the anti-ezrin antibody, however, the antibody was diluted 1:100 and pressure-cooking for $8 \mathrm{~min}$ was used for antigen retrieval.

10) Morphometric Measurements in immunostained tissues.

Immunostained glomeruli showed clear contours, which could be manually traced to measure the glomerular tuft area $\left(\mathrm{GTA}, \mu \mathrm{m}^{2}\right)$ of each glomerular midsection. Fifteen to 20 glomeruli midsections were measured for each rat. All morphometric measurements were done using the CAS 200 (Becton \& Dickinson Image Cytometry Systems, USA). Glomerular tuft volume (GTV) was estimated from the GTA and the measured section thickness, as described previously [6].

11) Ezrin positive area.

The glomerular tuft ezrin positive area (EzA) was measured using a threshold method and taken as an estimate of the total podocyte area. Podocyte ezrin positive area $\left(\mu m^{2}\right)$ was normalized to total glomerular capillary tuft area $\left(\mu m^{2}\right)$ or dividing the Ezrin positive area measured in each glomerular midsection by the number of podocyte nuclei observed in each mid-section. The threshold of stain intensity in each glomerular tuft section considered positive for ezrin was set at a level such that glomerular parietal cells showed clear and sharp brown stain and their whole cell body was visible. Parietal cells' ezrin stain did not differ in groups C, D, DI, DPG or DPLZ and served as internal control for each glomerulus.

12) Number of podocytes and podocyte density.

The number of nuclei within the glomerular tuft that stained positive for anti WT-1 was counted in each glomerular midsection. Fifteen to twenty glomeruli 
per section were counted per animal. The ezrin area per podocyte was calculated from the ratio of the total ezrin positive tuft area per glomerular midsection to the number of counted WT-1 positive nuclei per glomerular cross Section $(8,9)$. The total number of podocytes per glomerulus was derived from the number of WT-1 positive nuclei counted in each glomerular tuft midsection, the glomerular tuft area, the measured section thickness [9], and the glomerular tuft volume as described earlier [6].

Podocyte density was the ratio of calculated number of WT-1 positive nuclei per glomerulus to the glomerular tuft volume.

\section{3) Podocytes}

Podocytes were cultured in vitro as described before [10] [11]. Proteins were extracted after 7 or 10 days exposure to 5 or $25 \mathrm{mM}$ glucose and with or without 2.5 or $25 \mathrm{uM}$ PLZ. Western blots were done in the protein extracts of the cultures, using anti Ezrin antibody, were stained and scanned to measure the expression of Ezrin [6] [11]. Cultures in each codition were in triplicate.

14) Statistical Analysis

All results were expressed as mean $\pm \mathrm{SE}$ and were analyzed by one way ANOVA to establish differences between groups. When F statistics was significant, comparisons between any two groups was further tested using unpaired two-tailed students $t$-test for equal or unequal variances according to the equality of variance test. The software used was SPSS 11 for Windows. For all statistical tests, a $P$ value of less than 0.05 was considered significant.

\section{Results}

One week STZ diabetes caused a significant increase in blood glucose concentration, measured with Gluctrend, 2 Roche, $(26.39 \pm 1.17 \mathrm{mM}$, vs. $5.28 \pm 0.47 \mathrm{mM}$, $p<0.001)$ and protein excretion rate $(20.17 \pm 1.73 \mathrm{mg} / \mathrm{day}$, vs. $6.43 \pm 0.89$ $\mathrm{mg} /$ day, $p<0.001$ ), when compared to controls (Table 1 ). Treatment with PLZ reduced blood glucose concentration to $5.7 \pm 0.56 \mathrm{mM}$, near control levels, and prevented proteinuria $(8.97 \pm 1.07 \mathrm{mg} /$ day $)$. Insulin treatment also prevented the development of hyperglycemia $(5.14 \pm 0.37 \mathrm{mM})$ and proteinuria $(8.85 \pm 0.99$ $\mathrm{mg} /$ day).

1) Podocyte changes in STZ diabetes

There was a significant $(p<0.001)$ increase in immunostained GTA with diabetes (Figure 2, Table 2), confirming our earlier finding using PAS stain [6]. Glomerular hypertrophy was accompanied by a significant increase in immune-stained ezrin positive tuft area (EzA). The relative increase in EzA was higher than that of the glomerular tuft as indicated by the significant increase of the ratio of EzA to GTA (Table 2).

The number of WT-1 positive nuclei per glomerular tuft midsection area was significantly $(p<0.05)$ lower in diabetic rats compared to controls (Figure 1 , Table 3). However, the number of podocytes per glomerulus in one-week diabetic rats was not significantly different from their controls (Table 3 ). There were significant increases $(p<0.001)$ in ezrin area and volume $(P<0.05$ per 
Table 1. Blood glucose and protein excretion rate in all experimental groups.

\begin{tabular}{ccc}
\hline Group & BG $(\mathrm{mM})$ & PER $(\mathrm{mg} /$ day $)$ \\
\hline C $(n=12)$ & $5.28 \pm 0.47$ & $6.43 \pm 0.89$ \\
D $(n=7)$ & $26.39 \pm 1.17^{\star * *}$ & $20.17 \pm 1.73^{* * *}$ \\
DI $(n=11)$ & $5.14 \pm 0.37^{\mathrm{NS}}$ & $8.85 \pm 0.99^{\mathrm{NS}}$ \\
DPG $(n=10)$ & $30.32 \pm 1.67^{\star *}$ & $18.14 \pm 3.5^{\star}$ \\
DPLZ $(n=5)$ & $5.7 \pm 0.56^{\mathrm{NS}}$ & $8.97 \pm 1.07^{\mathrm{NS}}$ \\
\hline
\end{tabular}

Blood Glucose, $\mathrm{PER}=$ Protein excretion rate, $\mathrm{C}=$ control, $\mathrm{D}=$ Diabetic, $\mathrm{DPG}=$ Propylene glycol-treated diabetic, DPLZ $=$ Phlorizin-treated diabetic rats. Results are expressed as mean $\pm \mathrm{SE}$ and compared using unpaired two-tailed students't test. ${ }^{*}=p<0.05,{ }^{* *}=p<0.01,{ }^{* * *}=p<0.001$ compared to control.

Table 2. Glomerular ezrin positive area in insulin and phlorizin-treated diabetic rats.

\begin{tabular}{cccccc}
\hline & $\mathrm{C}$ & $\mathrm{D}$ & $\mathrm{DI}$ & DPG & DPLZ \\
& $\mathrm{n}=4, \mathrm{~N}=60$ & $\mathrm{n}=6, \mathrm{~N}=88$ & $\mathrm{n}=7, \mathrm{~N}=92$ & $\mathrm{n}=5, \mathrm{~N}=62$ & $\mathrm{n}=5, \mathrm{~N}=64$ \\
\hline GT um $^{2}$ & $5324 \pm 86$ & $5898.5 \pm 87.1^{* * *}$ & $5940.5 \pm 115^{* * *}$ & $6156.2 \pm 97.5$ & $6428.2 \pm 97.5^{* * *}$ \\
Ezrin Area um & & & & & \\
EzA/Tuft A \% & $512.4 \pm 10.6$ & $487.1 \pm 13.1^{* * *}$ & $474.5 \pm 17.9^{* * *}$ & $442.1 \pm 18.5^{*}$ & $360.2 \pm 12.8^{*}$ \\
\hline
\end{tabular}

$\mathrm{C}=$ Control, $\mathrm{D}=$ Diabetic, $\mathrm{DI}=$ Inulin-treated diabetic, DPG = Propylene glycol-treated diabetic, DPLZ = Phlorizin-treated diabetic rats, $\mathrm{n}=\mathrm{No}$. of rats, $\mathrm{N}=$ Total no. of observations. GTA = Glomerular tuft area, EzA $=$ Ezrin positive area. All variables were analyzed using one way ANOVA, and then compared using unpaired two-tailed student's $t$-test. ${ }^{*}=p 0.05,{ }^{* * *}=p<0.001$, compared to control. ${ }^{*}=p<0.05,{ }^{* * *}=p<$ 0.001 , compared to diabetic. $=p<0.001$, compared to DPG.

Table 3. Podocyte number, density and ezrin volume in insulin and phlorizin-treated diabetic rats.

\begin{tabular}{|c|c|c|c|c|c|}
\hline & $\begin{array}{c}\mathrm{C} \\
\mathrm{n}=4, \mathrm{~N}=60\end{array}$ & $\begin{array}{c}\mathrm{D} \\
\mathrm{n}=6, \mathrm{~N}=88\end{array}$ & $\begin{array}{c}\text { DI } \\
\mathrm{n}=7, \mathrm{~N}=92\end{array}$ & $\begin{array}{c}\text { DPG } \\
\mathrm{n}=5, \mathrm{~N}=62\end{array}$ & $\begin{array}{c}\text { DPLZ } \\
\mathrm{n}=5, \mathrm{~N}=64\end{array}$ \\
\hline $\begin{array}{l}\text { Pod no./ } \\
\text { glomerulus }\end{array}$ & $157.8 \pm 2.3$ & $153.2 \pm 1.8$ & $160.4 \pm 2.8$ & $160.7 \pm 2.6 \#$ & $158.4 \pm 4.5$ \\
\hline Pod no/GTV $\times 10^{6}$ & $327.3 \pm 4.7$ & $277.2 \pm 9^{* *}$ & $269.9 \pm 8.6^{\star *}$ & $271.7 \pm 11.5$ & $256.0 \pm 8.6^{\star * *}$ \\
\hline $\begin{array}{c}\text { Total ezrin }+ \text { ve } \\
\text { volume } \times 10^{3} \mathrm{um}^{3}\end{array}$ & $28.4 \pm 1.5$ & $47.75 \pm 2.5^{* * *}$ & $46.8 \pm 4.9^{*}$ & $42.0 \pm 3.7$ & $34.8 \pm 2.8$ \\
\hline $\begin{array}{c}\text { Ezrin +ve } \\
\text { volume/Pod um }\end{array}$ & $180.1 \pm 10.1$ & $298.6 \pm 16.3^{* * *}$ & $291.4 \pm 29.1^{*}$ & $262.2 \pm 25.1$ & $222.0 \pm 22.5$ \\
\hline
\end{tabular}

$\mathrm{C}=$ Control, $\mathrm{D}=$ Diabetic, $\mathrm{DPG}=$ Propylene glycol-treated diabetic, DPLZ = Phlorizin-treated diabetic rats. $\mathrm{n}=$ No. of rats, $\mathrm{N}=$ Total no. of observations. GTV = Glomerular tuft volume, Pod = Podocyte, Pod no/GTV = Podocyte density. Podocyte number was derived from Wilm's tumor-positive nuclei corrected for double counting, tissue loss and actual section thickness (9). Results are expressed as mean $\pm \mathrm{SE} .{ }^{* *}=p$ $<0.01,{ }^{* * *}=p<0.001$ compared to controls.

podocyte one week after the induction of diabetes (Table 4). Glomerular hypertrophy at one-week diabetes is accompanied by podocyte changes as indicated by the increases in EzA and ezrin area and volume per podocyte.

With the increases in GTV and the constant number of podocytes per glomerulus, the volume density of podocytes (number per unit GTV) was significantly $(p<0.01)$ lower at one week-diabetes than that in controls (Table 3$)$. 
CONTROL.

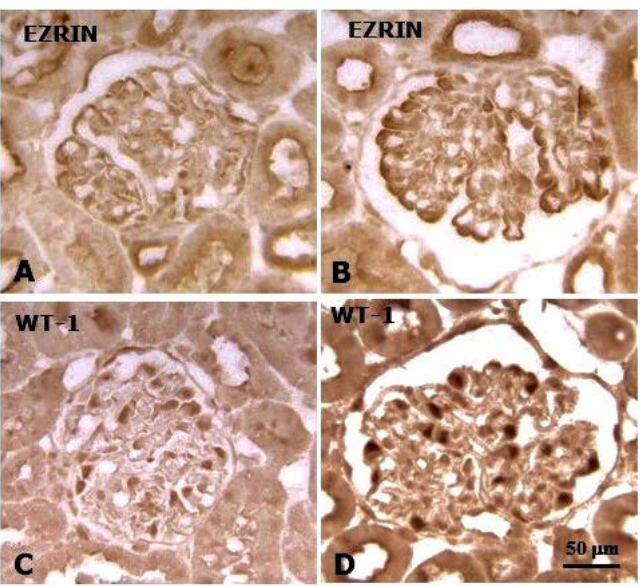

Figure 1. Glomerular morphological changes due to one-week diabetes in Fischer rats. There was an increase in ezrin-positive area in one-week diabetic rats (B) compared to controls (A). There was a decrease in the number of WT-1 positive nuclei per glomerular midsection in diabetic rats (D) when compared to controls (C), but no change in podocyte number per glomerulus after one week diabetes (see text) (A, B, C, D: $4 \mathrm{~m}$, paraffin sections, immunoperoxidase for anti-ezrin antibody $(\mathrm{A}, \mathrm{B})$ and for anti-WT-1 antibody $(\times 400)$.

Table 4. Glomerular podocytes in insulin- and phlorizin-treated diabetic rat.

\begin{tabular}{lccccc}
\hline & $\mathrm{C}$ & $\mathrm{D}$ & $\mathrm{DI}$ & $\mathrm{DPG}$ & $\mathrm{DPLZ}$ \\
$\mathrm{n}=4$ & $\mathrm{n}=6$ & $\mathrm{n}=5$ & $\mathrm{n}=5$ & $\mathrm{n}=5$ \\
\hline $\begin{array}{l}\text { Ezrin A um } \\
\text { Podocyte no. }\end{array}$ & $312.4 \pm 19.2$ & $486.9 \pm 30.8^{* *}$ & $461.6 \pm 50.3^{*}$ & $431.6 \pm 397.6$ & $357.7 \pm 27.4$ \\
$\begin{array}{l}\text { per GTA } \\
\text { EzA/Pod }\end{array}$ & $16.7 \pm 0.2$ & $15.52 \pm 0.3^{*}$ & $15.8 \pm 0.2^{*}$ & $15.9 \pm 0.4$ & $15.4 \pm 0.5^{\star}$ \\
\hline
\end{tabular}

EzA $=$ Ezrin positive area, GTA $=$ Glomerular tuft area. EzA/Pod $=$ Ezrin positive area per podocyte. $\mathrm{DI}=$ Inulin-treated diabetic, DPG = Propylene glycol-treated diabetic, DPLZ = Phlorizin-treated diabetic rats. All variables were analyzed using one way ANOVA, and then compared with control using unpaired two tailed student's $t$-test. ${ }^{*}=p 0.05,{ }^{*}=p<0.001$, compared to control. $=p<0.05$.

\section{2) Effect of phlorizin treatment on diabetic glomerular changes}

Phlorizin treatment significantly $(p<0.001)$ decreased the glomerular EzA (Figure 2, Table 2) compared with that observed in diabetic rats treated with PG (DPG group). However phlorizin did not completely prevent growth of EzA in diabetic rats, since EzA was still significantly $(p<0.05)$ larger in DPLZ than in control rats. However, EzA as percent of GTA (Table 2) and EzV per podocyte (Table 4) in the PLZ-treated diabetic group were not significantly different from those in controls. With phlorizin treatment, podocyte density per glomerulus was significantly $(p<0.001)$ lower than that in controls (Table 3$)$, since PLZ treatment did not alter podocyte number per glomerulus (Table 3 ) nor prevented glomerular tuft growth (Table 2).

3) Effect of insulin treatment on diabetic glomerular changes 
Insulin did not prevent the growth of glomerular tuft seen in one week diabetic rats: GTA and GTV were significantly $(p<0.001)$ larger in DI rats than in controls and were not different from those observed in untreated diabetic rats (Figure 1, Table $2 \&$ Table 3). In addition, insulin treatment did not prevent diabetes-induced podocyte changes. The ezrin positive glomerular area and its percent to total GTA were significantly $(p<0.001)$ higher in DI rats than in DPG controls and not different from those observed in untreated one-week diabetic rats (Figure 1, Table 2).

Estimated podocyte number per glomerulus in DI rats was not different from that in untreated diabetics or controls. This led to a significantly $(p<0.01)$ lower podocyte density per glomerular volume in DI rats than in controls, since their GTV were larger than controls (Table 3). The ezrin area/podocyte (Table 4) and the ezrin positive volume per podocyte (Table 3 ) in DI rats were significantly larger than in controls and not different from that in untreated diabetic rats. The podocyte changes with insulin or PLZ treatments are summarized in Figure 3.

4) Ezrin expression in cultured podocytes.

There were no differences in ezrin expression in triplicate western blots from protein extracs of podocytes grown for 7 (or 10) days in 25 or in $5 \mathrm{mM}$ glucose and with or without 2.5 or $25 \mathrm{uM} \mathrm{PLZ} \mathrm{(Figure} \mathrm{4).}$

\section{Discussion}

The close association of increases in ezrin positive area with hyperglycemia and its reversal on treatment with the hypoglycemic agent PLZ may be a direct one

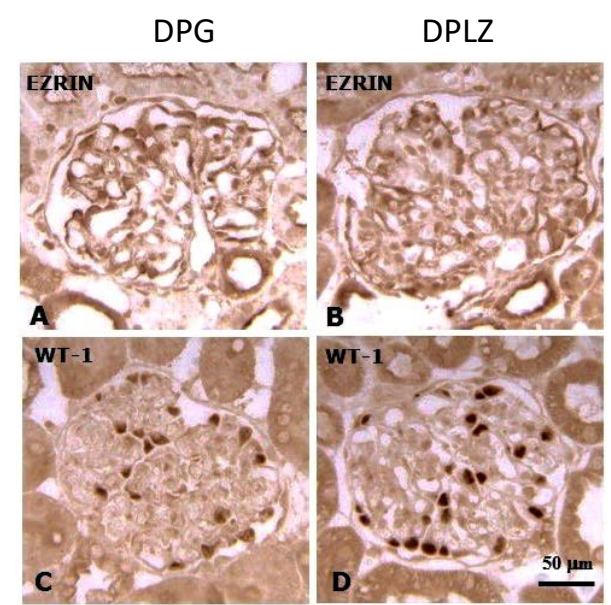

Figure 2. Glomerular morphological changes with one-week phlorizin treatment of diabetic Fischer rats (DPLZ). Ezrin expression was lower in DPLZ rats (B) than propylene glycol-treated diabetic rats (DPG) (A). There was no change in number of Wilm's tumor-1 (WT-1) positive nuclei per glomerular midsection in DPLZ rats (D) when compared with DPG (C). (A, B, C, D: 4 um, paraffin sections, immunoperoxidase for antiezrin antibody (A), (B) and for anti-WT-1 antibody (C), (D), ×400). 


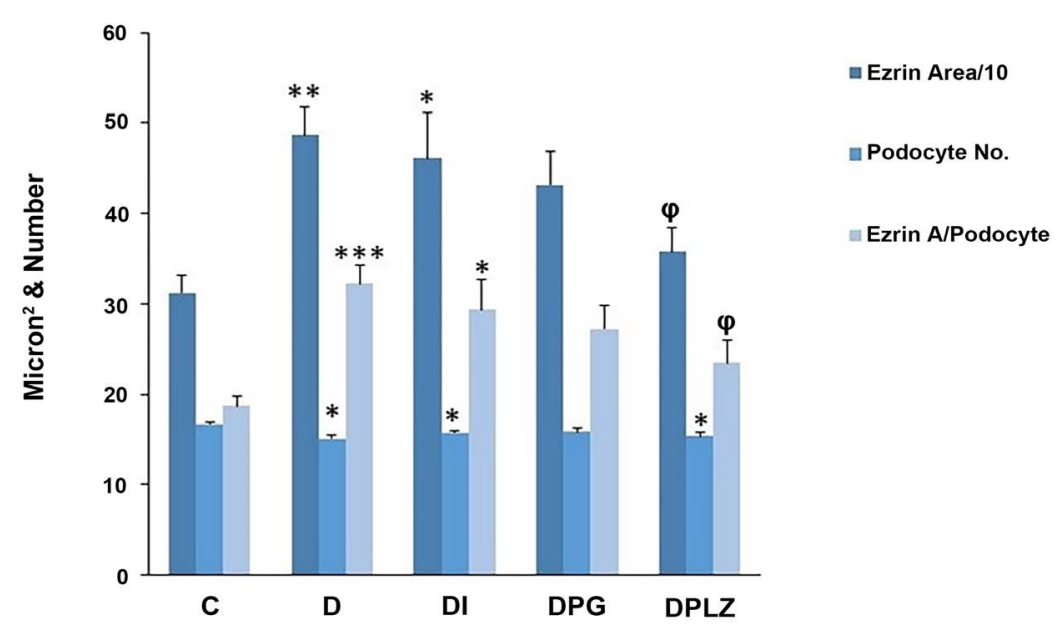

Figure 3. Glomerular podocyte changes in Diabetic (D), insulin (DI) or Phlorizin (DPLZ) treated diabetic, and Polyethylene glycol vehicle treated Diabetic (DPG) compared to control (C) rats. ${ }^{\star} p<0.05,{ }^{\star *} p<0.01,{ }^{* * *} p<0.001$ vs. C. $\varphi p<0.05$ vs. DPG.

7 days treatment $(n=3)$

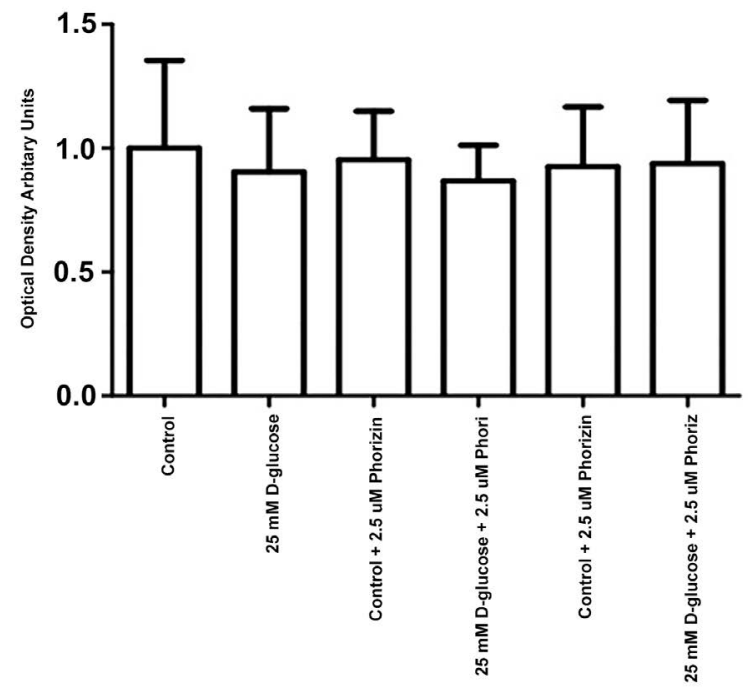

75

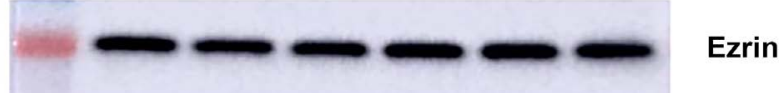

35

GAPDH

Figure 4. Expression of Ezrin and GDPH n podocyte cultures in vitro, exposed to 5 or $25 \mathrm{mM}$ glucose with and without 2, 5 or $25 \mathrm{uM} \mathrm{PLZ.}$

i.e. it may involve changes in glucose entry into podocytes. Podocytes express GLUT2, GLUT4 [10] and SGLT1, but not SGLT2 [11]. PLZ blocked 20\% of glucose uptake into rat podocytes in culture [10]. $80 \%$ of glucose entry is via facili- 
tated diffusion mainly through GLUT2. Glucose entry into podocytes triggers activation of various signaling pathways [12]. SGLT inhibitors may reduce glucose entry and this may be critical for the reversal of observed podocyte changes. Entry of STZ, used to induce diabetes may be reduced by PLZ and thus help to reduce oxidative stress that may cause the observed increase in glomerular ezrin positive area.

Alternatively increased ezrin expression may reflect an indirect effect of hyperglycemia (for example through changes in glomerular filtration or pressure) which is also reversed with PLZ treatment [6]. The relationship seems independent of insulin or C-peptide levels since PLZ reversed the changes but is not known to alter the blood levels of these peptides. Because increases in ezrin area also occur in other forms of glomerular hypertrophy such as that following $5 / 6$ nephrectomy [7] it is likely that the increase in ezrin positive area is a general response to podocyte stress rather than a specific effect of the elevated glucose level in uncontrolled diabetes. Indeed, insulin reduces blood glucose to normal but does not alter glomerular ezrin. In the subtotal nephrectomy model of glomerular growth and in diabetes, podocyte hypertrophy has been documented [13] and corresponds well in time with the increases in ezrin positive area that we observed in podocytes.

Changes in cross-linker protein ezrin may be related to changes in size, shape or cytoskeletal rearrangements in podocytes and its foot processes [7] that may be precursors to their later described effacement and rounding [14], altered integrin expression and basement membrane interactions [15] that can lead to their detachment and to membrane thickening.

Ezrin changes can also affect the distribution of charged membrane proteins such as podocalyxin in the filtration slits [14] or the structure of macromolecular complexes that influence the perm-selectivity of the slit diaphragms [15]. Insulin treatment did not prevent the podocyte changes (increased ezrin positive area, reduced density) observed early in diabetes but prevented the development of mild proteinuria. We have previously shown that proteinuria early in diabetes, is mainly related to hyperfiltration, which accounts for $60 \%$ of the variance in PER observed [6]. Both PLZ and insulin [6] [16] prevent hyperfiltration yet only PLZ prevents the increase in Ezrin positive area. PLZ affects filtration by normalizing tubulo-glomerular feedback [17]. Such signaling or metabolic effects of SGLT2 inhibitors [5] may be critical for reversal of increases in podocyte ezrin positive area by PLZ. By contrast, insulin promotes oxidative stress in podocytes [18] and this may contribute to the persistent increase in ezrin poitive area present in insulin-treated diabetic rats.

There was no overexpression of Ezrin in western blots of podocytes grown in culture with high compared to normal glucose, with or without PLZ suggesting that the Ezrin changes observed in glomeruli of STZ rats are not related to high glucose and suggesting that they may reflect changes in the cell distribution of Ezrin in STZ diabetes, prevented by PLZ binding to its membrane receptors. However we did not measure the amounts of Ezrin protein expression in podo- 
cytes in vivo.

In diabetes of more prolonged duration, decreases rather than increases in Ezrin areas in the glomeruli has been found associated with podocyte effacement, detachment, basement membrane thickening and overt proteinuria [19]. Decreases in ezrin expression also occur in kidneys of children with severe proteinuria nephrotic syndrome [20], particularly in steroid resistant cases. Since ezrin is a cytoskeletal cross-linker, it is not surprising that rearrangements of the membrane-cytoskeletal structures in the podocytes occur with changes in actin associated proteins [21] and that excess or deficits of ezrin are associated with changes in permeability of the glomerular filtration barrier. Early in diabetes, when there is increase in ezrin positive area, the proteinuria is mild and related to hyperfiltration [6]. By contrast, later in diabetes and in nephrotic syndromes with severe proteinuria, severe podocyte detachment and reduced expression of ezrin occur $(20,21)$. PLZ and other SGLT-inhibitors that specifically reverse increases in podocyte ezrin area may be useful to prevent podocyte detachment and disease progression.

Reduced glomerular podocyte density observed early in diabetes was entirely due to growth of the glomerular capillary tuft which persisted with PLZ treatment despite normalization of glycemia, of the ezrin area per podocyte and the reduction in proteinuria. Thus the reduced glomerular podocyte density observed is not responsible for the mild proteinuria seen early in diabetes. In more advanced disease when podocyte density is severely reduced due to loss of podocytes from the glomeruli [20], low podocyte density contributes more to proteinuria [21]. Persistent glomerular tuft growth early in diabetes, despite normal EzA per podocyte and normal mesangial matrix area [6] with PLZ treatment, indicates that it is due to growth/enlargement of capillary endothelial cells. Tuft growth is not due to dilation of capillaries since PLZ treatment normalizes GFR [6] but not tuft growth. Podocytes secrete vascular endothelial growth factor VEGF [22] and stromal cells derived growth factor [23]. Capillary endothelial cells have the receptors for these growth factors [23]. VEGF is elevated in diabetic nephropathy and blocking it improves renal structure and function [24]. Tuft growth is not due to lack of insulin since insulin treatment does not prevent it [25] [26]. Persistent lack of C-peptide despite PLZ or insulin treatments can contribute to glomerular capillary tuft growth since its replacement is known to prevent glomerular hypertrophy [27]. C-peptide signaling occurs through a G protein coupled receptor, PLC, L-type $\mathrm{Ca}^{2+}$ channels, PKC, and ERK to control cell proliferation and angiogenesis [27] [28]. Glomerular tuft growth may be important for progression of disease to glomerular sclerosis as some cases are known to evolve without proteinuria or very low GFR [29]. In conclusion, this study shows that the sodium-glucose transport inhibitor phlorizin but not insulin, reduces redistribution of ezrin to the surface of glomerular podocytes of STZ rats, early in diabetes, contributing to slow morphological changes and progression of nephropathy. 


\section{Acknowledgements}

We thank the Departments of Physiology and Pathology of Kuwait University and the Department of Pediatric Nephrology at the University of Bristol for financial support.

\section{Conflicts of Interest}

The authors declare no conflicts of interest regarding the publication of this paper.

\section{References}

[1] Pavenstädt, H. (2000) Roles of the Podocyte in Glomerular Function. American Journal of Physiology, 278, F173-F179. https://doi.org/10.1152/ajprenal.2000.278.2.F173

[2] Reiser, J. and Sever, S. (2013) Podocyte Biology and Pathogenesis of Kidney Disease. Annual Review of Medicine, 64, 357-366. https://doi.org/10.1146/annurev-med-050311-163340

[3] Christiansen, J.S., Gammelgaard, J., Frandsen, M., et al. (1981) Increased Kidney Size, Glomerular Filtration Rate and Renal Plasma Flow in Short-Term Insulin-Dependent Diabetics. Diabetologia, 20, 451-456. https://doi.org/10.1007/BF00253406

[4] Yee, J. (2008) Diabetic Kidney Disease: Chronic Kidney Disease and Diabetes. Diabetes Spectrum, 21, 8-10. https://doi.org/10.2337/diaspect.21.1.8

[5] Clar, C., Gill, J.A., Court, R., et al. (2012) Systematic Review of SGLT2 Receptor Inhibitors in Dual or Triple Therapy in Type 2 Diabetes. BMJ Open, 2, e001007. https://doi.org/10.1136/bmjopen-2012-001007

[6] Malatiali, S., Francis, I. and Barac-Nieto, M. (2008) Phlorizin Prevents Glomerular Hyperfiltration But Not Hypertrophy in Diabetic Rats. Experimental Diabetes Research, 2008, Article ID: 305403. https://doi.org/10.1155/2008/305403

[7] Hugo, C., Nangaku, M., Shakland, S.J., et al. (1998) The Plasma Membrane-Actin Linking Protein, Ezrin, Is a Glomerular Epithelial Cell Marker in Glomerulogenesis, in the Adult Kidney and in Glomerular Injury. Kidney International, 54, 1934-1944. https://doi.org/10.1046/j.1523-1755.1998.00195.x

[8] Kim, Y.H., Goyal, M., Kurnit, D., et al. (2001) Podocyte Depletion and Glomerulosclerosis Have a Direct Relationship in the PAN-Treated Rat. Kidney International, 60, 957-968. https://doi.org/10.1046/j.1523-1755.2001.060003957.x

[9] Sanden, S.K., Wiggins, J.E., Goyal, M., et al. (2003) Evaluation of a Thick and Thin Section Method for Estimation of Podocyte Number, Glomerular Volume, and Glomerular Volume per Podocyte in Rat Kidney with Wilms' Tumor-1 Protein Used as a Podocyte Nuclear Marker. Journal of the American Society of Nephrology, 14, 2484-2493. https://doi.org/10.1097/01.ASN.0000089829.45296.7C

[10] Lewko, B., Bryl, E., Witkowski, J.M., et al. (2005) Characterization of Glucose Uptake by Cultured Rat Podocytes. Kidney and Blood Pressure Research, 28, 1-7.

[11] Saleem, M.A., O’Hare, M.J., Reiser, J., Coward, R.J., Inward, C.D., Farren, T., Xing, C.Y., Ni, L., Mathieson, P.W. and Mundel, P. (2002) A Conditionally Immortalized Human Podocyte Cell Line Demonstrating Nephrin and Podocin Expression Jour nal of the American Society of Nephrology, 13, i630-i638. 
[12] Tabatabai, N.M., North, P.E., Regner, K.R., et al. (2014) De Novo Expression of Sodium-Glucose Cotransporter SGLT2 in Bowman's Capsule Coincides with Replacement of Parietal Epithelial Cell Layer with Proximal Tubule-Like Epithelium. The Journal of Membrane Biology, 247, 675-683. https://doi.org/10.1007/s00232-014-9686-4

[13] Li, C. and Siragy, H.M. (2014) High Glucose Induces Podocyte Injury via Enhanced (Pro) Renin Receptor-Wnt-b-Catenin-Snail Signaling Pathway. PLOS ONE, 9, e89233. https://doi.org/10.1371/journal.pone.0089233

[14] Lee, G.S.L., Nast, C.C., Peng, S.C., et al. (1998) Differential Response of Glomerular Epithelial and Mesangial Cells after Subtotal Nephrectomy. Kidney International, 53, 1389-1398. https://doi.org/10.1046/j.1523-1755.1998.00871.x

[15] Wegner, B., Al-Momany, A., Kulak, S.C., et al. (2010) CLIC5A, a Component of the Ezrin-Podocalyxin Complex in Glomeruli, Is a Determinant of Podocyte Integrity. American Journal of Physiology-Renal Physiology, 298, F1492-F1503. https://doi.org/10.1152/ajprenal.00030.2010

[16] Perisic, L., Rodriguez, P.Q., Hultenby, K., et al. (2015) Schip1 Is a Novel Podocyte Foot Process Protein That Mediates Actin Cytoskeleton Rearrangements and Forms a Complex with Nherf2 and Ezrin. PLoS ONE, 10, e0122067. https://doi.org/10.1371/journal.pone.0122067

[17] Malatiali, S., Francis, I. and Barac-Nieto, M. (2017) Insulin Prevents Hyperfiltration and Proteinuria But Not Glomerular Hypertrophy and Increases Mesangial Matrix Expansion in Diabetic Rats. Medical Principles and Practice, 26, 78-83. https://doi.org/10.1159/000450864

[18] Vallon, V. (2011) The Proximal Tubule in the Pathophysiology of the Diabetic Kidney. American Journal of Physiology-Regulatory, Integrative and Comparative Physiology, 300, R1009-R1022. https://doi.org/10.1152/ajpregu.00809.2010

[19] Kim, E.Y., Anderson, M. and Dryer, S.E. (2012) Insulin Increases Surface Expression of TRPC6 Channels in Podocytes: Role of NADPH Oxidases and Reactive Oxygen Species. American Journal of Physiology-Renal Physiology, 302, F298-F307. https://doi.org/10.1152/ajprenal.00423.2011

[20] Wasik, A.A., Koskelainen, S., Hyvönen, M.E., et al. (2014) Ezrin Is Down-Regulated in Diabetic Kidney Glomeruli and Regulates Actin Reorganization and Glucose Uptake via GLUT1 in Cultured Podocytes. The American Journal of Pathology, 184, 1727-1739. https://doi.org/10.1016/j.ajpath.2014.03.002

[21] Ostalska-Nowicka, D., Zachwieja, J., Nowick, M., Kaczmarek, E., et al. (2006) Ezrin-A Useful Factor in the Prognosis of Nephrotic Syndrome in Children: An Immunohistochemical Approach. Journal of Clinical Pathology, 59, 916-920. https://doi.org/10.1136/jcp.2005.031732

[22] White, K.E. and Bilous, R.W. (2004) Diabiopsies Study Group: Structural Alterations to the Podocyte Are Related to Proteinuria in Type 2 Diabetic Patients. Nephrology Dialysis Transplantation, 19, 1437-1440. https://doi.org/10.1093/ndt/gfh129

[23] Tufo, A. and Veron, D. (2012) VEGF and Podocytes in Diabetic Nephropathy. Seminars in Nephrology, 32, 385-393. https://doi.org/10.1016/j.semnephrol.2012.06.010

[24] Sayyed, S.G., Hägele, H., Kulkarni, O.P., et al. (2009) Podocytes Produce Homeostatic Chemokine Stromal Cell-Derived Factor-1/CXCL12, Which Contributes to Glomerulosclerosis, Podocyte Loss and Albuminuria in a Mouse Model of Type 2 Diabetes. Diabetologia, 52, 2445-2454. https://doi.org/10.1007/s00125-009-1493-6

[25] Schrijvers, B.F., Flyvbjerg, A. and De Vriese, A.S. (2004) The Role of Vascular En- 
dothelial Growth Factor (VEGF) in Renal Pathophysiology. Kidney International, 65, 2003-2017. https://doi.org/10.1111/j.1523-1755.2004.00621.x

[26] Carraro, M., Mancini, W., Artero, M., et al. (2000) Albumin Permeability in Isolated Glomeruli in Incipient Experimental Diabetes Mellitus. Diabetologia, 43, 235-241. https://doi.org/10.1007/s001250050035

[27] Samnegard, B., Jacobson, S.H., Jaremko, G., et al. (2001) Effects of C-Peptide on Glomerular and Renal Size and Renal Function in Diabetic Rats. Kidney International, 60, 1258-1265. https://doi.org/10.1046/j.1523-1755.2001.00964.x

[28] Walcher, D., Babiak, C., Poletek, P., et al. (2006) C-Peptide Induces Vascular Smooth Muscle Cell Proliferation. Involvement of Src-Kinase, Phosphatidylinositol 3-Kinase, and Extracellular Signal-Regulated Kinase 1/2. Circulation Research, 99, 1181-1187. https://doi.org/10.1161/01.RES.0000251231.16993.88

[29] MacIsaac, R.J., Tsalamandris, C., Panagiotopoulos, S., et al. (2004) Non-Albuminuric Renal Insufficiency in Type 2 Diabetes. Diabetes Care, 27, 195-200.

https://doi.org/10.2337/diacare.27.1.195 